\title{
The Predictive Value of Pre-Implant Pulmonary Function Testing in LVAD Patients
}

\author{
Nicholas Hess ${ }^{1}$, Laura Seese ${ }^{2}$, Gavin Hickey², Mary Keebler ${ }^{2}$, Yisi Wang ${ }^{2}$, Floyd Thoma ${ }^{2}$, \\ and Arman Kilic ${ }^{2}$ \\ ${ }^{1}$ University of Pittsburgh Medical Center \\ ${ }^{2}$ University of Pittsburgh Medical Center Health System
}

August 20, 2020

\begin{abstract}
Background: The predictive value of preoperative pulmonary function testing (PFT) in left ventricular assist device (LVAD) patients remains unknown. This study evaluates the relationship between abnormal PFTs and postimplant outcomes in LVAD patients. Methods: LVAD implants from January 2004 to December 2018 at a single institution were included. Patients were stratified based on presence of abnormal preoperative PFTs, and the primary outcome was respiratory adverse events (AE). Secondary outcomes included one-year overall postimplant survival, and complications including bleeding, renal failure, thromboembolism, and device malfunction. Results: 333 patients underwent LVAD implant, 46.5\% (n=155) with normal PFTs and $53.5 \%(\mathrm{n}=178)$ with abnormal PFTs. Patients with abnormal PFTs were noted to have higher rates of respiratory AEs $(25.9 \%$ vs $15.1 \%, \mathrm{p}=0.049)$. In multivariable analysis, the impact of PFTs was most significant when FEV1/FVC ratio was $<0.5$ (HR 16.32, 95\% CI 1.70, 156.78). The rates of other AEs including bleeding, renal failure, right heart failure, and device malfunction were similar. One-year overall postimplant survival was comparable between the groups $(56.8 \%$ vs $68.8 \%$, $\mathrm{p}=0.3183)$, though patients in the lowest strata of FEV1 $(<60 \%$ predicted $)$ and FEV1/FVC $(<0.5)$ had elevated risk-adjusted hazards for mortality (HR 2.63 95\% CI 1.51, 4.60 and HR 18.92, 95\% CI 2.10, 170.40, respectively). Conclusions: The presence of abnormal preoperative PFTs is not prohibitory for LVAD implantation although it can be used for risk stratification for respiratory AEs and mortality, particularly in patients with severely reduced metrics. The importance of careful patient selection should be underscored in this higher risk patient subset.
\end{abstract}

\section{Hosted file}

LVAD_PFTs_JOCS_8_19_20_flat.doc available at https://authorea.com/users/331307/articles/ 476784-the-predictive-value-of-pre-implant-pulmonary-function-testing-in-lvad-patients 


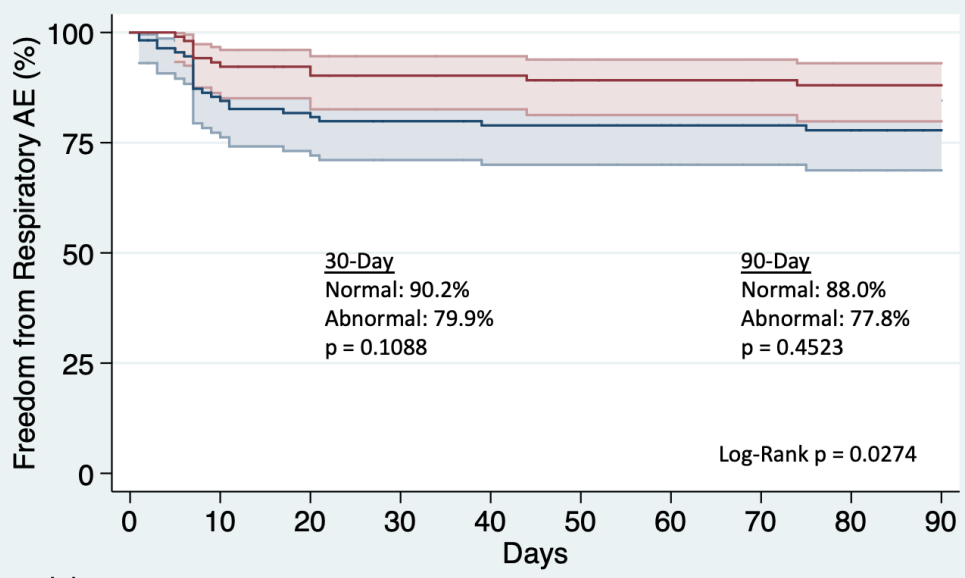

Number at risk

Abnormal 11

Normal 106

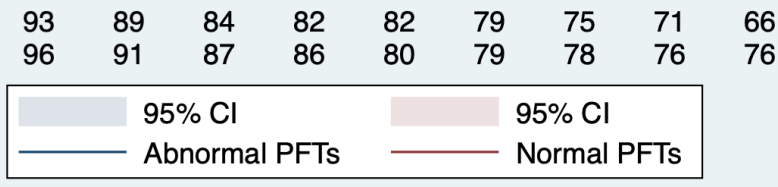

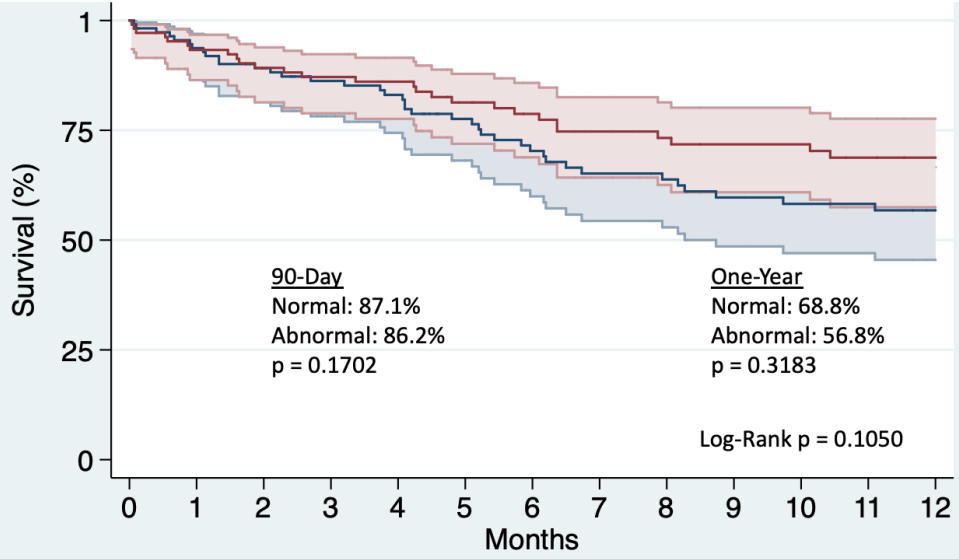

Number at risk

$\begin{array}{llllllllllllll}\text { Abnormal } & 112 & 104 & 95 & 83 & 77 & 66 & 56 & 49 & 47 & 43 & 40 & 40 & 37\end{array}$ $\begin{array}{llllllllllllll}\text { Normal } & 106 & 95 & 86 & 83 & 77 & 65 & 59 & 53 & 50 & 48 & 48 & 44 & 42\end{array}$

$95 \% \mathrm{Cl}$

$95 \% \mathrm{Cl}$

Abnormal PFTs

Normal PFTs 